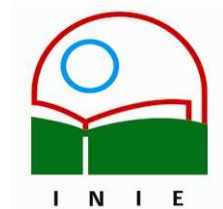

Actualidades Investigativas en Educación

Revista Electrónica publicada por el

Instituto de Investigación en Educación

Universidad de Costa Rica

ISSN 1409-4703

http://revista.inie.ucr.ac.cr

COSTA RICA

\title{
LA EDUCACIÓN DEL FUTURO, EL FUTURO DE LA EDUCACIÓN EN MÉXICO
}

THE EDUCATION OF THE FUTURE, THE FUTURE OF EDUCATION IN MEXICO

\author{
Volumen 8, Número 1 \\ pp. 1-33
}

Este número se publicó el 30 de abril 2008

José Guadalupe Vargas Hernández

La revista está indexada en los directorios:

LATINDEX $, \underline{\text { REDALYC}}, \underline{\text { IRESIE}}, \underline{\text { CLASE}}$, DIALNET, DOAJ, E-REVIST@S,

La revista está incluida en los sitios:

REDIE, RINACE, OEI, MAESTROTECA, HUASCARAN 


\title{
LA EDUCACIÓN DEL FUTURO, EL FUTURO DE LA EDUCACIÓN EN MÉXICO
}

\author{
THE EDUCATION OF THE FUTURE, THE FUTURE OF EDUCATION IN MEXICO
}

\section{José Guadalupe Vargas Hernández ${ }^{1}$}

\begin{abstract}
Resumen: Este trabajo tiene como propósito analizar la prospectiva de la educación en México bajo un horizonte del año 2020 a partir de la consideración de los principales cambios del entorno económico, político y social. La hipótesis central del análisis se fundamenta en que el logro de las metas de calidad, cobertura y pertinencia proyectadas para la atención de la educación superior, generación, difusión y aplicación del conocimiento científico, tecnológico, cultural y humano está determinada por las formas de gobernanza institucional del sector educativo, científico y tecnológico y por la apertura y contribuciones de nuevas fuentes de financiamiento. El método de investigación empleado se centró principalmente en el análisis de las tendencias de formulación de estrategias y políticas. Las implicaciones económicas y políticas de las transformaciones educativas obligan a repensar y redefinir la función de la educación superior y a agendar en los próximos años las actividades de los procesos de transformación institucional con su impacto en el crecimiento económico y el desarrollo social. Los desafíos que tiene la educación superior implican repensar su papel y misión a fin de establecer las estrategias alternativas para el futuro en un marco de desarrollo sustentable, cultura de paz y una ética global.
\end{abstract}

Palabras clave: EDUCACIÓN SUPERIOR, MÉXICO, POLÍTICA EDUCATIVA, PROSPECTIVA EDUCATIVA.

Abstract: This paper has the purpose to analyze of the prospective in education in Mexico under a horizon of the year 2020, beginning from the consideration of the main changes in the economic, political and social environment. The main hypothesis of this analysis is based on the goals achievement of projected quality, coverage and pertinence for the attention of higher education, generation, diffusion and implementation of scientific, technological, human and cultural knowledge are determined by the institutional governance forms of the educative, scientific and technological sector and by the openness and contributions of new financial sources. The research method used was centered mainly on the analysis of tendencies of strategies and policies formulation. The economic and political implications of the educative transformation oblige to re think its role and mission to the end of establishing the alternative strategies for the future under a framework of sustainable development, culture of peace and global ethics.

Key words: HIGHER EDUCATION, MEXICO, EDUCATIVE POLICY, EDUCATIVE PROSPECTIVE.

\begin{abstract}
1 Doctor en Administración Pública (Columbia States University), Doctor en Economía (Keele University, England). Otros estudios de Doctorado en Comportamiento Organizacional (Lancaster University). Maestro en Administración de Empresas con especialidad en Dirección Industrial (Pacific States University). Marketing Certificate (The British Institute of Marketing). Profesor investigador miembro del Sistema Nacional de Investigadores, Nivel 1, Visiting Scholar, University of California-Berkeley. Profesor del Instituto Tecnológico de Cd. Guzmán. Profesor investigador del Doctorado Interinstitucional en Administración, Universidad Autónoma de San Luís Potosí, México.
\end{abstract}

Direcciones electrónicas: jvargas2006@gmail.com, igvh0811@yahoo.com

Artículo recibido: 23 de enero, 2008

Aprobado: 15 de abril, 2008 


\section{Introducción}

Este trabajo tiene como propósito analizar la prospectiva de la educación en México bajo un horizonte del año 2020 a partir del la consideración de los principales cambios del entorno económico, político y social. Con este enfoque, se establecen como ejes del análisis: la gobernanza de la educación, la política de la educación superior, la ciencia y la tecnología, el proyecto de la institución de educación superior, ciencia y tecnología del futuro, la generación e innovación del conocimiento, la vinculación, las finanzas y la evaluación integral del proceso educativo. Finalmente se hace una prospectiva de las tareas pendientes de la educación superior en México, y se asume un análisis crítico sobre los alcances de las funciones del nuevo modelo de las instituciones de educación superior (IES) bajo una orientación prioritaria a los requerimientos del mercado, subestimando las necesidades sociales y las del Estado mexicano.

\section{Entorno}

El nivel de educación alcanzado por los diferentes pueblos del mundo divide a las naciones entre aquellos que solo un pequeño segmento ha logrado los beneficios y aquellos que están alcanzando grandes avances al conjugar una distribución más equitativa de oportunidades de formación y educación con mayor rendimiento. Las economías menos desarrolladas no cuentan con un sistema educativo eficiente para formar el suficiente capital humano con la capacidad para incorporar el conocimiento en todos los sectores de la economía.

La educación superior en México debe intentar responder a los patrones internacionales y la dinámica de los procesos de globalización económica. Estos procesos de globalización más propios de las economías post industriales, arrastrarán consigo tendencias hacia la organización posmoderna y la sociedad del conocimiento para apuntalar un proyecto hegemónico de sociedad global que dé sustento al mercado global y a una democracia global. La sociedad del conocimiento más parece una utopía cuya realidad implica que las Instituciones de Educación Superior (IES) se transformen bajo un guion de libre mercado como el imperativo de un pensamiento hegemónico cada vez más confrontado por las manifestaciones de la diversidad cultural.

En este esquema, la función de las IES es la generación y aplicación del conocimiento que sustente el orden social y el crecimiento económico mediante la producción de saberes científicos, tecnológicos y culturales y de la gestión del talento humano capaz de generar una 
economía dinámica, integrada y competitiva. Sin embargo, uno de los principales retos que enfrentarán las IES será la de dar respuestas concretas a una sociedad más allá de considerarla como una simple entidad abstracta con capacidad de consumo. La competencia en los sistemas de educación superior estará cada vez más globalizada, por lo que se requiere transformar los procesos institucionales. Las implicaciones de la pertinencia de la educación superior en un mundo bajo procesos de globalización económica están delimitadas por los requerimientos de competencias necesarias para realizar las actividades del mercado global, los idiomas y los sistemas de comunicación e información, entro otros.

Las IES tendrán procesos de transformaciones profundas en los próximos años, aunque lamentablemente bajo un esquema de modernización occidental centrado en los modelos euro-anglo-norteamericanos. Con estas transformaciones se estrecharán cada vez más los espacios para reconocer y analizar las consecuencias de las transformaciones profundas que suponen la economía global, la cybersociedad y la posmodernidad organizacional como forma para superar las burocracias institucionales (Ibarra Colado, 2007). Como consecuencia de estas transformaciones, surgirán nuevos actores y un nuevo modelo de gestión en las universidades. La dinámica de los procesos de globalización económica que pone en riesgo las relaciones interpersonales en todas las formas de convivencia social, requiere como imperativo una revisión moral de las responsabilidades de los diferentes actores, desde una ética global. Las IES en México no deben descuidar en los próximos años la preservación del capital social y acrecentarlo con planes y programas para resolver los problemas ancestrales del desarrollo y, sobre todo, superar las confrontaciones y conflictos que conllevan las nuevas realidades.

La globalización de la educación superior tiene consecuencias perjudiciales y beneficiosas en la educación superior, como por ejemplo, la falta de regularización de universidades, planes de estudio de baja calidad, etc. En la consolidación de las IES entra como un factor importante a considerar la nueva división internacional del trabajo universitario, especialmente en la producción de ciencia y tecnología y en la preparación de cuadros altos de científicos para la producción de conocimientos de punta. La consolidación de esta nueva división internacional del trabajo universitario es el resultado de la generación y aplicación de conocimientos científicos y tecnológicos de frontera para aquellas instituciones de educación superior del mundo más desarrollado, mientras que, para el caso de México, los países menos desarrollados todavía seguirán siendo los consumidores de estos conocimientos que 
se generan en otras latitudes y con otras realidades. De allí que la transferencia tecnológica y la innovación tendrá un papel preponderante en nuestras IES. Resulta un desafío imperativo la promoción y el impulso del desarrollo, además de la utilización de las nuevas tecnologías de la información y la comunicación para insertar los procesos de generación y aplicación de los conocimientos científicos y tecnológicos en la sociedad de la información y del conocimiento.

La denominada sociedad de la información y el conocimiento es una consecuencia de los procesos de globalización y del avance de las tecnologías de la información y las comunicaciones (TICs). La transformación de la educación superior debe centrarse en generar, investigar, organizar, coordinar, compartir, difundir y aplicar el conocimiento en el gran contexto de la sociedad global del conocimiento y dar respuesta a las crecientes demandas del desarrollo. Todas las IES deberán estar conectadas en redes administrativas, académicas y científicas, logrando la conectividad total de los procesos. No obstante, se deberá tener plena conciencia de la posible dependencia tecnológica, el factor de costobeneficio y las bondades que pueden ofrecer las TICs.

Se presentan muchas semejanzas en las tendencias en la educación superior a nivel global, tales como la universalización de la educación superior, la cual se expresa en una diversificación de la oferta educativa con diferentes opciones de planes y programas de estudio. El cambio de los contenidos de planes y programas de estudio debe permitir a los egresados de las IES mayores oportunidades para insertarse en el mercado laboral, considerando los requerimientos de la especialización dados los avances científicos y tecnológicos y la formación transdisciplinaria (Morin, 1999a, 1999b, 1999c; Congreso Internacional, 1997; y Nicolescu, 1997). La transdisciplinariedad, la complejidad de las IES y la incertidumbre de su entorno más globalizado constituyen el nuevo paradigma que va más allá del mero enfoque economicista y que considera a la realidad cada vez más compleja e interrelacionada, incierta e impredecible.

La transformación de los contenidos en un diseño curricular de planes y programas de estudio lleva implícita una revisión de los principios de la filosofía educativa y el rediseño de los modelos académicos y de organización institucional, los nuevos perfiles de egreso, la retícula, estrategias pedagógicas capaces de impulsar la transdisciplinariedad y la complejidad de los fenómenos científicos y tecnológicos. Una realidad cada vez más 
compleja presenta como retos a la IES de los próximos años, la necesidad de integrar más que descomponer, los procesos de generación y aplicación del conocimientito y, por lo tanto, el desafío de diseñar las estructuras más eficaces y eficientes. Además de que las sociedades generan y transmiten la información y los conocimientos, aquellas que se incorporan a la economía del conocimiento, registran, comercializan, importan, exportan y aplican el conocimiento, eliminando las barreras tradicionales entre las manufacturas y los servicios. Estas economías basadas en el conocimiento son más competitivas debido al valor agregado que ofrecen los sistemas de investigación e innovación científica y tecnológica.

No obstante, los rápidos avances científicos, sobre todo de las tecnologías de la información y la comunicación, aunado a los crecientes procesos de globalización económica, hacen necesario revisar y replantear estos supuestos como un requerimiento de una política de Estado en educación superior pública, ciencia y tecnología, en una perspectiva estructural y de largo aliento, si se quiere dar respuesta a las crecientes demandas de la sociedad mexicana del siglo XXI. El supuesto del modelo neoliberal de que el Estado-nación ya no requiere de la acción legitimadora de las IES, se modifica para que las IES se centren en la realización de las funciones de generación, difusión y aplicación del conocimiento y a la formación de profesionales que el mercado global y la sociedad global demanden. El Estado mexicano debe tener siempre presente que su principal función es la rectoría de la educación superior y mantener los espacios necesarios para la formulación e implementación de las políticas educativa y para la toma de decisiones, por encima de cualquier interés mediático de otros grupos de poder con intereses en la educación superior, de tal forma que no deje lugar a cuestionamientos su responsabilidad de función de Estado y el control de la calidad de los procesos educativos.

En la lógica de las relaciones Estado - sociedad - empresas - IES (Educación Superior) se presenta una gran tensión en la racionalidad gubernamental (Casanova, 2002) centrada en las necesidades sociales y la racionalidad instrumental dirigida hacia la eficiencia de la economía y la rentabilidad del mercado. Los intereses del sector empresarial se presentan mediante diversas formas organizacionales para reclamar su participación en el control de las IES en un mercado educativo. Acosta Silva, (2000) argumenta que los procesos de liberalización económica y política desarrollados en buena parte de las sociedades latinoamericanas contemporáneas, parecen hallar sentido en el marco de una redefinición de las fronteras de la acción pública y de la resignificación de la acción pública misma. A una 
larga tradición estatalista de la acción pública le ha seguido una tendencia liberadora y civilista de los asuntos públicos, más asociada a los mecanismos del mercado y a la dinámica de los equilibrios democratizadores provocada por las fuerzas políticas y sociales emergentes...

Las reformas estructurales de la educación superior en México tienen su principal sustento teórico en la modernización bajo un entorno de procesos de globalización económica. Esas reformas estructurales de la educación superior tienen como finalidad superar las crisis de cobertura de la demanda educativa (Ibarra, 2002). La implementación de las reformas en las IES presupone la determinación de ciertos principios teóricos, técnicos y políticos. El crecimiento de la cobertura en educación está supeditada a políticas de contratación e inducción de académicos de alto rendimiento y de alto nivel de calidad académica. Estas reformas orientadas a un nuevo proyecto de institucionalización académica, implican reformas en sus estructuras, en los diseños institucionales, en la gobernabilidad institucional y de diferenciación funcional.

La transformación del papel del Estado implica procesos de reingeniería para el diseño de las nuevas funciones en materia de educación, investigación y desarrollo tecnológico. El diseño organizacional de las IES implica una transformación de estructuras, mecanismos y comportamientos participativos para la cooperación en las comunidades académicas, así como también la modificación de la normatividad y formas de gobernabilidad institucional bajo nuevos esquemas de gestión del talento humano y relaciones laborales. El Estado mexicano deberá seguir manteniendo la regulación activa de las IES mediante el fortalecimiento de los mecanismos del mercado y la promoción de la vinculación de la empresa para estimular la producción académica y científica.

Sin embargo, el papel que desempeñan los diferentes actores en la regulación, operación y coordinación de los sistemas de educación superior, requieren transformaciones en las estructuras, procesos y comportamientos, de tal forma que se facilite la implementación de estrategias en un medio ambiente externo. Las estrategias de transformación institucional de la educación superior deberán ser consensuadas entre los principales actores y grupos de interés, entre los que destacan las organizaciones empresariales e industriales, el Sindicato Nacional de Trabajadores de la Educación, las asociaciones de padres de familia, etc. Herrera (2002) puntualiza que la urgencia de la transformación institucional de la educación 
superior radica en la convergencia de fuerzas tales como la tendencia internacional determinada por el impacto de la globalización y por la revolución científico-tecnológica del final del siglo XX. El planteamiento de políticas de educación nacionales que muestran una gran receptividad a las recomendaciones de organismos multilaterales de procesos de búsqueda, en la mayoría de los casos, provocan reformas que conducen a una mayor calidad, cobertura y pertinencia dentro de la concepción tradicional, disciplinaria, burocrática y jerárquica en nuestro país.

La transformación institucional deberá propiciar un cambio en el modelo educativo de tal forma que implique el cuestionamiento del modelo de sociedad en el que se fundamenta y que conduzca a las universidades a otros modos de pensar, de organizar, de producir y transmitir los saberes (Lanz, 2003). El futuro de las generaciones mexicanas ya está predeterminado por un conjunto de políticas neoliberales bajo una visión de procesos de integración global. El escenario del componente económico más viable es el tendencial con continuidad en los actuales niveles de incertidumbre, crisis eventual y tasas de crecimiento económico moderado. Con respecto al mercado laboral y el empleo, se prevé un crecimiento acelerado del sector de los servicios con una mayor población ocupada en este segmento, y un menor crecimiento de los sectores industrial y el primario (ANUIES, 1999). La tendencia a la terciarización de la economía en una sociedad centrada en la información y el conocimiento, tendrá un impacto en los perfiles de formación científica y tecnológica profesional (ANUIES, 1999). Los cambios económicos en el escenario global tienen serias repercusiones en el mercado laboral y esto en los cambios de las fronteras del conocimiento. Los cambios en el entorno presionan para la transformación del sistema de educación superior. La Asociación Nacional de Universidades e Instituciones de Educación Superior (ANUIES) realizó el diagnóstico de la problemática de la educación superior en México en una prospectiva para el 2020 con una propuesta de acciones inmediatas para impulsar el sistema de educación superior. Se reconocen rezagos en todo el sector de la educación superior, déficit en la calidad de la educación, bajos niveles de logro académico, infraestructura insuficiente y tecnológicamente obsoleta, deficiencias en la prestación de servicios a sectores vulnerables de la sociedad mexicana, como por ejemplo la población indígena, el poco interés por la calidad educativa, etc. (ANUIES, 1999).

En materia de desarrollo nacional y regional y el papel del Estado, el escenario del 2020 es un modelo de concertación y coordinación flexible y descentralizada con un sistema estatal 
de innovación e interacción entre empresas, mercado, gobiernos y universidades. Este modelo de gobernabilidad interinstitucional debe superar al actual modelo de la racionalidad instrumental de los mercados que pone a funcionar las instituciones sociales bajo esquemas de productividad orientados a maximizar beneficios individuales de los diferentes actores que bajo una concepción voluntarista actúan con oportunismo en detrimento de los objetivos del desarrollo social y la solidaridad.

Los gobiernos deben seguir garantizando el cumplimiento del derecho a la educación superior y asumiendo la responsabilidad de su financiamiento. El escenario del sistema político que se prevé será capaz de generar una gobernabilidad democrática y una política social amplia y sistemática, que considere la dimensión de las desigualdades económicas y sociales de México. La reducción de las desigualdades regionales con respecto a los conglomerados sociales y de género, para alcanzar las oportunidades educativas solo se logra si se amplía la cobertura y se establecen políticas y programas compensatorios para los grupos sociales más vulnerables. La desigualdad en el acceso a la educación superior es todavía un asunto pendiente, sobre todo en la distribución territorial de oportunidades de educación superior, en donde según datos del Instituto Nacional de Estadística, Geografía e Informática (INEGI) el Sur y Sureste de México tienen menos oportunidades educativas que el Centro y el Norte (INEGI, 2000). Se tiene que avanzar de forma sustancial en lo referente a las nuevas responsabilidades en materia de educación e investigación del Estado y de los particulares en la transformación de la educación superior. En el componente demográfico, las proyecciones del Consejo Nacional de Población (CONAPO) indican que el crecimiento demográfico tenderá a disminuir en las próximas cinco décadas: para el 2010 la población oscilaría entre 111.3 millones de habitantes y 116; para el 2020 en 119.7 a 130.5; para el 2030 de 124.4 a 142.2 y para el 2050 de 122 a 152.2 (p. 15). El crecimiento de la población demandante de educación superior, de 15 a 64 años, aumentaría hasta alcanzar los 80.8 millones en el 2031 para luego descender progresivamente.

Para el grupo de edad específico de educación superior (de 16 a 22 años) se prevé que crecerá hasta el 2010 para descender a partir del 2025, lo cual implica que habrá que responder con la creación de nuevas universidades. Un crecimiento urbano desordenado tendrá consecuencias desfavorables en las IES. La dinámica demográfica apuntala el reto de la cobertura de la educación superior, la cual dificulta la mejora de la calidad. No debe 
asumirse que de la posición que ocupan las IES se deriva la calidad educativa debido a la existencia de procesos de auto selección y factores estructurales (ANUIES, 1999).

Las IES tienen que prevenir en un futuro inmediato la seguridad con su entorno mediante acciones de vigilancia y prevención de conductas ilegales e indeseables. En un futuro, las IES tendrán la obligación de proyectarse como instituciones más saludables con programas como Universidad Saludable, lo cual implica la sanidad del entorno universitario o tecnológico y de todos los actores involucrados en los procesos de generación y aplicación de conocimientos, implantados a través de programas de formación de valores, comportamientos y actitudes de ética global, cultura de paz y desarrollo sustentable del medio ambiente, determinación de acciones de prevención para atención de actores que tipifiquen delitos o que simplemente se identifiquen con perfiles de violencia, drogadicción, y otras conductas que pongan en riesgo la integridad de los demás actores.

\section{Gobernanza en la educación}

La transformación de los modelos de gobierno y organización de las IES lleva implícito una revisión profunda de las normas jurídicas para sustentar las modificaciones estructurales, de comportamientos y de procesos tecnológicos. Es por ahora un desafío imperativo realizar una revisión profunda a las normas del sistema educativo nacional en lo general y en particular a todo el marco regulatorio de las IES. Los poderes locales, municipales y estatales, desempeñan un papel importante en los procesos de cambio estructural mediante la implementación de las políticas educativas promovidas para favorecer la transformación de las IES. Las instituciones de educación superior deben ampliar los cauces democráticos y definir claramente las facultades y funciones limitadas. Es necesario diseñar instituciones de educación superior que integren el sistema educativo nacional y que atiendan de manera diversificada, flexible y en forma pertinente a grupos sociales que han sido tradicionalmente excluidos de la posibilidad de obtener una educación de alto nivel y de buena calidad. Las IES de los próximos años continuarán jugando un papel social importante que dará espacio a los nuevos agregados sociales emergentes para confrontar sus paradigmas, ideologías y estructuras de poder.

Las reformas institucionales al sistema de educación superior en México requieren de estrategias de alcance, escala, cobertura y profundidad en los procesos de cambio institucional. Las reformas de las instituciones de educación superior seguirán 
reorientándose mediante los criterios de calidad, pertinencia e internacionalización. La forma de gobierno debe estar centrada en la solución de los problemas de sus funciones sustantivas, las necesidades institucionales para generar y desarrollar el conocimiento científico y tecnológico como su principal razón de ser o misión, mediante el uso más eficiente y eficaz de los recursos disponibles. Los gobiernos de las IES deben fomentar los mecanismos democráticos de consulta tendientes a sumar esfuerzos en las tareas sustantivas, establecer las normas del gobierno y los requisitos de acceso al mismo.

El control corporativo de los trabajadores de la IES continuará perdiendo terreno en los próximos años a pesar de las tendencias señaladas por Analítica Consultores (2006), en el sentido de que las fuerzas corporativas representada por el Sindicato Nacional de Trabajadores de la Educación, lucharán por mantener sus privilegios frente a dos partidos políticos, el Partido Revolucionario Institucional (PRI) y el Partido Acción Nacional (PAN) para "colonizan" a los sistemas educativos estatales. El fortalecimiento de la vida democrática de las IES debe iniciarse con el fortalecimiento de las formas de gobierno orientadas por los cuerpos colegiados que regulen las actividades académicas y de investigación, financiamiento, etc. La creación de las formas de gobierno y organización de las IES, si bien centradas en la eficiencia económica, con mayor eficacia en el desarrollo social, seguirá siendo uno de los grandes retos, especialmente porque se requieren nuevas estrategias para la generación y aplicación del conocimiento cuando están cambiando los paradigmas de la educación científica y tecnológica superior. Las nuevas formas de gobierno y legislación de las IES tienen que ser coherentes con los retos de los procesos de globalización económica y con la revolución de las tecnologías de la información y las comunicaciones.

Es un imperativo para los próximos años establecer sistemas de registro, selección e inducción de aspirantes a puestos directivos en las IES, lo que implica la asignación por concurso de cargos directivos. Así mismo, los procesos de toma de decisiones deben abrirse a procesos más democráticos, mediante el fortalecimiento de estructuras más planas, uso de tecnologías de información y comunicación de punta y comportamientos sujetos a procesos de transparencia y rendición de cuentas. La diversificación de las estructuras institucionales se manifiesta en una diversidad de formas organizacionales para dar atención a las necesidades de educación, ciencia y tecnología mediante programas y planes de estudio. Los procesos de toma de decisiones deben ser transparentes, horizontales e 
incluyentes que ofrezcan la apertura a todos los actores involucrados, pero sobre todo a los grupos docente, investigador y estudiantil, los principales implicados en los procesos de enseñanza-aprendizaje. La apertura tiene que permitir nuevas formas de elección del gobierno de las IES, de tal forma que se promueva una mayor participación en toda la comunidad académica y científica. Se propone la apertura de espacios para el ejercicio de la democracia académica bajo un principio de relación igualitaria estudiantes-docentes (incluye docentes e investigadores-investigadoras) - administración-gobierno.

La búsqueda de alternativas conlleva a la construcción de espacios educativos innovadores que permitan la expresión abierta, flexible, transdisciplinaria y autorregulada de la vida académica de la multiplicidad de actores. La autonomía universitaria de una gran variedad de instituciones de educación superior, ciencia y tecnología, hacen más significativas las relaciones de coordinación interinstitucional para aprovechar las ventajas comparativas y las áreas de excelencia para compartir recursos, conjuntar esfuerzos y programas a través de alianzas estratégicas. Avances en las alianzas estratégicas y de programas internacionales interinstitucionales para complementar las infraestructuras para docencia, investigación y posgrado, de tal forma que se propicie una mayor movilidad académica estudiantil, docente e investigadora. La vida académica debe estar sustentada en relaciones horizontales con la parte administrativa. En el futuro se debe estar más preocupado porque las formas de gobierno de las IES, sus estructuras sean representativas de los principales actores del proceso de enseñanza aprendizaje y de investigación, y en general el involucramiento en los procesos de toma de decisiones de los principales beneficiarios la comunidad, los negocios y la sociedad civil. La creación y mantenimiento de redes académicas estatales, regionales, nacionales e internacionales contribuirán a hacer más eficiente el uso de los recursos para ofrecer servicios educativos innovadores, de gran calidad, cobertura y pertinencia. La calidad de la educación es entendida en forma multidimensional por comprender la calidad de docencia, gestión, programas académicos, estudiantes, etc. El Plan Nacional de Desarrollo (2006-2012) entiende por calidad del sistema educativo, una variable multidimensional que conjuga acceso, equidad, pertinencia y resultados educativos. Este plan enuncia estrategias de carácter sectorial y transversal para fortalecer los procesos de evaluación del aprendizaje, el desempeño docente y la gestión institucional.

El mejoramiento sustancial de la calidad de la educación superior debe sustentarse en el mejoramiento de los estudios de posgrado de docentes, y en el ejercicio del Sistema 
Nacional de Evaluación y Acreditación. Se pretende que para el año 2020 se encuentre consolidado el sistema nacional de planeación y de evaluación, acreditación y aseguramiento de la calidad de la educación superior. Mejorar los sistemas de planeación, coordinación, control, evaluación y acreditación de programas e instituciones de todo el sistema educativo mexicano es un imperativo urgente. Los supuestos sobre los cuales funciona el actual modelo de educación superior en México, los mecanismos de regulación gubernamental y de un arreglo institucional centrado en la racionalidad económica han conducido a una severa crisis. Si bien estos supuestos son lógicamente coherentes durante la fase de formulación, su operabilidad no es viable en la realidad contingente del contexto mexicano (Meyer y Rowan, 1999).

Concretamente, lo que se propone en la racionalidad del proyecto neoliberal es la reconfiguración de la gobernabilidad institucional de la nueva universidad bajo mecanismos de regulación definidos por el esquema de economía cuyas exigencias están siendo orientadas por procesos de integración global. En el caso de México, la tendencia a la regulación gubernamental y al ordenamiento institucional modificará profundamente las relaciones entre el Estado mexicano, las organizaciones del mercado y las IES. La regulación gubernamental se sustenta en un sistema de financiamiento sujeto a exigencias institucionales y a procesos de evaluación por resultados. En parte, la respuesta se fundamenta en los procesos de formulación de la política para el sector de la educación superior, la ciencia y la tecnología nacional.

\section{La política de la educación superior, la ciencia y la tecnología}

En México, la política social actual se propone reducir los niveles de pobreza y desigualdad económica y social a través de acciones y programas que tiendan a igualar el acceso a mejores estándares de educación, salud, vivienda y nutrición. La política educativa tiene que ser el instrumento que direccione las tendencias actuales en las cuales se debe implementar la transformación de la educación superior, de tal forma que se dé respuesta a los retos de los tiempos inciertos y complejos por venir. Los principios de relevancia, calidad e internacionalización, de acuerdo con la UNESCO, deben guiar los continuos cambios en la educación superior. Por relevancia se entiende el papel y sitio que ocupa la educación superior en la sociedad y en el mercado laboral, las relaciones con el Estado y el financiamiento público. 
La calidad se considera en forma multidimensional en sus productos, procesos del sistema educativo, personal académico, programas, estudiantes, infraestructura, entorno interno y externo, cultura de la evaluación, de la regulación y la autonomía, responsabilidad y rendición de cuentas, etc., que deberá funcionar como un todo coherente para garantizar la pertinencia social. La pertinencia de la educación superior tenderá a profundizar los procesos de diferenciación y segmentación en la dinámica del mercado laboral mundial. El principio de internacionalización deberá dar sustento a la movilidad e intercambios académicos orientados a un mayor entendimiento entre las culturas y una mayor difusión del conocimiento. Las instituciones educativas dan diferentes respuestas al cambio en un contexto institucional en función de sus capacidades para procesar las políticas educativas nacionales, debido en parte a la articulación de los diferentes actores y grupos de interés que participan en el diseño institucional con visiones diferentes.

Las líneas de la política educativa muestran una tendencia a la continuidad y profundización en el Plan Nacional de Desarrollo (PND) 2007-2012, el cual propone una educación basada en una transformación educativa, con pocas aportaciones para superar las limitaciones del sistema educativo nacional (Observatorio Ciudadano de la Educación, 2007b). Bajo el concepto de desarrollo humano, el PND se propone asegurar a la población mexicana la satisfacción de las necesidades de educación (PND, 200-2012, p. 23) y reconoce como propuesta para el Proyecto México 2030, lograr una educación de calidad con formación de valores y capacidades. La promoción del desarrollo humano sustentable se orienta por un perfeccionamiento centrado en la educación y la formación como elementos esenciales.

Los procesos de transformación institucional de la educación superior en México involucran a poderosos grupos de intereses en los niveles local y federal en la formulación e implementación de estrategias, por lo que se ha demostrado que cada institución es un caso diferente. El conjunto de planes gubernamentales que intentan modificar el sistema de educación superior muestran características distintas de implementación, si se considera la voluntad de los actores y autonomía de las instituciones públicas y privadas. Una de las principales tendencias en los sistemas de educación superior es la expansión cuantitativa que permita el acceso de todos los grupos sociales en las regiones. En un futuro próximo, el sistema educativo mexicano debe terminar la marcada desigualdad de la distribución geográfica. 
Es imprescindible ampliar las opciones educativas disponibles a todas las mexicanas y mexicanos independientemente de que se pretenda beneficiar a las fundamentalmente rurales o indígenas en determinadas regiones del país. La cobertura de la educación superior tiene serias deudas con los pueblos indígenas de México, por los contrastes en materia de oportunidades educativas que se ofrecen a los diferentes conglomerados sociales. Por lo tanto, es un imperativo para los próximos años la promoción de un modelo educativo que matice las oportunidades por regiones y culturas de tal forma que exista una mejor distribución de oportunidades para la calidad y equidad de estas culturas. La transformación educativa es uno de los retos de la igualdad de oportunidades propuesta en el desarrollo programático del PND (2006-2012). Las prioridades se resumen en la expansión general del sistema para garantizar oportunidades educativas a toda la población, eliminar la exclusión económica, informatizar el sistema educativo nacional, coordinar y descentralizar y apoyar a la docencia mediante recursos y formación (Observatorio Ciudadano de la Educación, 2007, p. 34).

Las estrategias para la educación se ubican en los ejes de la economía competitiva y generadora de empleos, igualdad de oportunidades y sustentabilidad ambiental. En la línea de "crecimiento elevado de la productividad", las políticas sugeridas conectan la educación y el desarrollo científico y tecnológico con la productividad, los cuales poco abonan para incrementar los avances ya que han estado limitados por las inversiones tanto del sector público como del privado. En el último sexenio (2000-2006) la tendencia fue decreciente en la proporción del gasto y el PIB para el desarrollo de la investigación y tecnología.

Hasta ahora, las aportaciones de la iniciativa privada han sido irrelevantes si se compara con otros países donde realizan copiosas contribuciones por ser los principales beneficiarios de la investigación en ciencia y tecnología. La educación privada superior debe continuar siendo flexible para adaptarse a las condiciones futuras de los cambios de la gobernabilidad entre las agencias reguladoras de los mercados - la sociedad - y los tres niveles de gobierno. La función de las IES históricamente reclamada de ser el valuarte cultural en las sociedades modernas, se está convirtiendo en uno más de los proveedores de servicios educativos al servicio de las leyes del libre mercado. En el sector privado se encuentran instituciones de educación superior de élite que atienden a estudiantes de muy alto niveles de ingresos económicos y con altos niveles de calidad académica por un lado, y por el otro, aquellas universidades que reciben los excedentes de la demanda no atendida, las cuales no 
necesariamente alcanzan la calidad promedio de las IES y que también se caracterizan por módicas cuotas de colegiaturas. Quizás el paso más importante que debe dar la educación privada es lograr la descentralización territorial para incrementar su capacidad de respuesta a las comunidades, localidades y municipios. La fuerte orientación hacia el mercado no debe ser motivo de descuido de aquellas actividades que por las fuertes inversiones en su estructura, no son de rentabilidad inmediata (Altbch, 2002).

Entre los supuestos de la educación pública superior en México, esta se asume como uno de los compromisos sociales del Estado con las características de gratuita, universal y laica. Es una responsabilidad de la sociedad mexicana demandar que el Estado cumpla con sus funciones para hacer viable en una dimensión de largo plazo, el proyecto de educación superior que impulse y logre la concertación de todas las voluntades en un pacto amplio e incluyente de todos los sectores sociales. El Estado mexicano continuará garantizando y haciendo efectivo el derecho social de acceso a la educación pública a todos los ciudadanos que elijan continuarla en alguna de las instituciones de educación superior, ciencia y tecnología, ya sea pública o privada. En los próximos años resulta un imperativo el establecimiento de sistemas y mecanismos para que los ciudadanos puedan realizar propuestas de mejoras, activar los consejos de participación social previstos ya en la Ley General de Educación, dando cauce a los reclamos de más participación ciudadana y reconociendo con esto a los grupos de interés que se contraponen al estatismo de la educación. Las IES tienen que estar abiertas a la participación de los diferentes grupos de interés a través de procedimientos que puedan materializar sus mejores intenciones.

La proyección social del desenvolvimiento presente y futuro de las IES debe orientarse por políticas educativas más coherentes con las responsabilidades que surgen de la realidad económica, política y social de nuestro pueblo; es decir, políticas educativas con una orientación plena de política social. La sociedad mexicana del siglo XXI deposita en las instituciones promotoras del conocimiento ciertas responsabilidades y expectativas a las cuales las instituciones de educación superior (IES), universidades y tecnológicos, no pueden mantenerse ajenas (Casanova Cardiel, 2007, p. 36). Para lograr lo anterior, en los próximos años se tiene la visión de un proyecto de reforma estructural nacional de la educación superior, ciencia y tecnología, impulsado por el Estado y con una fuerte participación de los sectores productivos y sociales. Este proyecto debe ser capaz de impulsar a futuro un nuevo modelo de desarrollo nacional, y considerar las asimetrías y 
desigualdades que existen en los diferentes contextos económicos, sociales y culturales en que se provee la educación superior, la ciencia y la tecnología.

Alcanzar una mayor equidad en la distribución del ingreso en un país como México donde hay una gran desigualdad en este rubro, representa un reto que está relacionado con el crecimiento económico y, por tanto, el crecimiento de la oferta educativa para ampliar la cobertura de oportunidades. Las políticas de mayor equidad que tengan un impacto educativo dependen de las finanzas y de mecanismos para evitar los riesgos de la segmentación social. El proyecto educativo nacional debe garantizar la equidad educativa a través de mecanismos diseñados por el federalismo educativo, pero además debe equilibrar esta equidad con el involucramiento de la cultura escolar a las culturas de las comunidades, de tal forma que fortalezca una educación intercultural. La promoción de la equidad educativa atenderá a poblaciones objetivo mediante la implementación de estrategias transversales de políticas sociales para apoyar a quienes más lo requieran (Observatorio Ciudadano de la Educación, 2007). El nuevo proyecto educativo está estrechamente vinculado al proyecto de nación que se desea para México, y debe ser el resultado de una política del Estado sustentada en la calidad y la competitividad del sistema educativo mexicano.

La política de la educación superior, ciencia y tecnología, requiere ser reformulada y rearticulada de forma integral, por tener de referente y finalidad la mejora de la educación nacional. La promoción de una educación integral implica las relaciones de cooperación y conflicto entre las IES, las comunidades, empresas, nuevos movimientos sociales y los diferentes niveles de gobierno. La educación integral también implica la promoción del desarrollo humano, los valores morales y cívicos, el desarrollo sustentable, una cultura de paz, la educación física y artística, etc. Una política de Estado en materia de educación superior, ciencia y tecnología, ha de promover la modernización de las instituciones encargadas de proveer este servicio y fortalecer la calidad, la transparencia la rendición de cuentas. Las políticas de educación superior, ciencia y tecnología, deben fijarse con el objetivo de incrementar sustancialmente la cobertura de la educación superior, abierta a un mayor alcance de atención que supere el $50 \%$ de la población total de jóvenes, de tal forma que se haga realidad uno de los grandes principios del Estado mexicano. Esto es factible si se considera la ventana de oportunidad poblacional debido a la tendencia en las bajas de la natalidad ya presente en la última década (INEGI, 2000). 
Las políticas educativas que se propongan deben ser en función de dar respuesta a los desafíos de las principales demandas a las IES para atender los requerimientos de cobertura, calidad y pertinencia, así como las actividades adecuadas para llevarlas a cabo (Rodríguez, 1998). Las políticas de transformación educativa deben ser capaces de convocar y aglutinar a los diferentes grupos de interés bajo una misma orientación teóricoepistemológica y metodológica para aumentar los niveles de cooperación, desalentar la competencia disfuncional centrada en el individualismo, disminuir el sentimiento de pérdida de autonomía institucional y promover la participación y articulación de los cuerpos y las comunidades académicas (Didriksson, 2002, pp. 368-370). Se tienen que instrumentar políticas educativas orientadas a resolver integralmente el problema de la deserción académica del y de la estudiante, sobre todo con acciones preventivas, tales como las tutorías académicas, a aquellos que pueden ser más propensos a incurrir en índices de bajo aprovechamiento académico. Se hace urgente la implementación de un sistema integrado de atención a los y las estudiantes que facilite los trámites interinstitucionales para una mayor movilidad académica y para todo tipo de apoyo académico y de servicios estudiantiles, lo cual se establece en un proyecto de institución superior, ciencia y tecnología.

\section{Proyecto de Institución de Educación superior, ciencia y tecnología en el futuro}

La educación superior, ciencia y tecnología en México es entregada a la sociedad por una gran diversidad de instituciones de educación superior, ciencia y tecnología. Toda institución de educación superior (IES), universidad o tecnológico, es por definición abierta a la sociedad. Todos los componentes institucionales del sistema de educación superior, como un conjunto plural, deberán actuar para lograr los objetivos propuestos a largo plazo. La construcción de este nuevo orden institucional basado en "la diferenciación, las jerarquías formalizadas y la coordinación burocrática" es un orden modernizador, "que estimula a los individuos y no a las corporaciones, donde se intenta someter la política interna a los códigos formales de la cultura académica" (Acosta Silva, 2000, p. 314). Las políticas educativas articuladas con las reformas institucionales implican la transición hacia nuevos modelos de desarrollo de las IES que requieren de nuevos procesos de coordinación y gestión bajo estructuras y comportamientos más flexibles y menos burocráticos, alejados de las jerarquías autoritarias. 
Se impone una revisión profunda de las IES para su transformación, con la participación de los diversos sectores involucrados, especialmente los sectores externos. El diseño del nuevo sistema educativo nacional tiene que abandonar su posición de formador de recursos humanos destinados a las maquiladoras para trascender el consumo de conocimientos científicos y tecnológicos típico de las sociedades que permanecen en los márgenes del subdesarrollo. En el caso de México, hasta ahora los profesionales encuentran trabajo en las plantas maquiladoras para las actividades técnicas de operación pero no para el diseño y la innovación de procesos y sistemas. La transformación de la educación superior tiene que centrarse en consideraciones del análisis del mercado laboral futuro y sus requerimientos de la formación profesional. Esto implica una perspectiva transdisciplinaria de los conocimientos, habilidades y destrezas científicas y tecnológicas que implican diseños curriculares complejos, en función de los compromisos para contribuir al desarrollo de la sociedad mexicana. El diseño institucional de las IES del futuro se inscribirá en un marco de estructuras, procesos y comportamientos que resultarán de las presiones de las tendencias ambientales complejas e inciertas y a la fortaleza de sus competencias, recursos y capacidades propias. El diseño de institución de educación superior de los próximos años es el de una organización compleja y diferenciada, orientada a la organización para la generación y aplicación de conocimientos transdisciplinarios, bajo nuevos esquemas de procesos de aprendizaje e investigación para ser transferidos a la sociedad.

Barnett (2002, p. 142) sugiere que

En este contexto, las ideas de impredicibilidad, desafío, ignorancia, turbulencia, riesgo, complejidad, inestabilidad e incluso caos, serán conceptos alrededor de los cuales es posible repensar la universidad. No hay forma de evitar esta situación, de modo que tenemos que afrontarla, pues en lo más profundo de nuestra conciencia universitaria sabemos que la educación superior no puede continuar como está.

En el plano epistemológico, el diseño institucional de las IES se debatirá entre las filosofías utilitarias, individualistas y de libre competencia en un extremo, y las filosofías sociales centradas en las formas solidarias de las finalidades sustantivas que implican compromisos de colaboración entre los diferentes actores implicados en la generación del conocimiento. Las nuevas formas de generación y aplicación del conocimiento requieren del diseño y reinvención de nuevas formas organizacionales que desencadenan innovadores modos de existencia de los actores y agentes locales con profundas y graves consecuencias sociales. 
Las IES deben ser una universidad o un tecnológico con un proyecto social que trascienda hacia afuera en todas las esferas del desarrollo humano. La complejidad institucional del sistema educativo nacional se expresa en el respeto a la autonomía de las universidades que dificulta lograr acuerdos tendientes a complementar recursos.

Por lo tanto, se requiere de una reforma institucional y su consolidación sobre una estructura que facilite la coordinación y complementación racional y equitativa de recursos y esfuerzos de las instituciones de educación superior, ciencia y tecnología para alcanzar niveles más altos de desarrollo que atiendan los perfiles regionales. El debate se centrará en el diseño de un modelo de universidad más orientado al sentido utilitario de la enseñanza y la investigación que en el diseño de un modelo de universidad con un proyecto utópico de emancipación democrática. Las IES implementarán programas de mejoras en la cantidad y calidad de su infraestructura, condiciones ambientales y programas académicos, con un impulso paralelo a acciones de evaluación de sus indicadores. Los criterios de la calidad, certificación y acreditación de programas académicos serán establecidos por cuerpos académicos consolidados en las instituciones de educación superior, ciencia y tecnología. La calidad académica de los programas de educación superior, ciencia y tecnología, será la condición para incrementar los procesos de certificación y para orientar el crecimiento cuantitativo.

Los modelos, proyectos y programas de educación superior asumirán mayor compromiso que en la actualidad, por un enfoque integral de la calidad que implique la participación de todos los actores de los procesos educativos para la calidad educativa, al igual que la calidad de sus procesos de administración de la educación (ANUIES, 1999). El modelo de educación superior, ciencia y tecnología que se proponga, tiene que reconocer la importancia de los factores del proceso de enseñanza aprendizaje en los resultados medidos en términos de indicadores de calidad y pertinencia. La transformación institucional debe profundizar las innovaciones en el modelo académico que tome en consideración la emergencia de los procesos de globalización económica, los cambios científicos y tecnológicos más avanzados en las diferentes áreas del conocimiento, el avance de las tecnologías de la comunicación e información.

La ANUIES (1999) recomienda seguir las estrategias de planeamiento y coordinación para asegurar la integración del sistema de educación superior. Todas estas tareas se realizarán 
con calidad, pertinencia, equidad y cantidad equiparables con los estándares internacionales. Las estrategias para la implementación de la transformación institucional en educación superior deberán considerar la multiplicidad de variables implicadas. Entre estas variables implicadas se destacan las políticas educativas nacionales y la madurez y capacidad de negociación de los actores institucionales. En el año 2020, las IES de México integrarán un sistema de educación superior capaz de formar profesionales e investigadores de alto nivel, generar y aplicar conocimientos, extender y preservar la cultura. A continuación se analizará con más detalle en que consistirán los procesos de generación e innovación del conocimiento.

\section{Generación e innovación del conocimiento}

El modelo educativo del futuro de la educación superior en México debe tener una sustentación transdisciplinaria, centrado en el desarrollo humano, congruente con una identidad cultural promotora de la paz, orientado por valores de una ética global y como estrategia para que nuestra sociedad alcance altos niveles de bienestar y desarrollo sustentable. En los próximos años será un imperativo generar y aplicar el conocimiento para atender los problemas ambientales generados por prácticas humanas que atentan contra su misma existencia. El modelo de formación profesional que hoy se mantiene fragmentado (ANUIES, 1999) en sus campos de conocimiento científico y tecnológico, aspirará en los próximos años a ser más integral, más humano, más completo, más multidimensional y sobre todo con una visión holista.

Si bien se debe enfatizar la estructura curricular que permita una mejor formación profesional para el mercado laboral, con la adopción del modelo curricular por competencias y estructuras modulares, no se debe menospreciar la formación integral del ser humano, considerado como persona y como ciudadano que tiene que convivir en un sistema social, político y cultural. Los diferentes actores del proceso educativo en el futuro tienen que ser capaces de generar e innovar el conocimiento con un enfoque de formación holista, más que reproducirlo como una condición básica para promover un desarrollo interdependiente en nuestros pueblos. Estos actores deberán favorecer y fortalecer las redes de comunicación y difusión científica y tecnológica internacionales. La formación profesional del futuro debe centrar más la atención en la enseñanza de los valores. La formación profesional debe ser promotora de los valores más humanos, que reconozca la centralidad del y de la estudiante pero que desaliente el individualismo egoísta, que fomente el respeto a la vida en sociedad, 
sin descuidar la solidaridad social, la conservación de la naturaleza y el medio ambiente. El modelo educativo tiene que considerar las diferencias individuales en los procesos de aprendizaje y contar con el apoyo del y de la docente para organizar y compartir sus experiencias mediante procesos académicos. Con respecto a los procesos académicos, se debe desarrollar la tendencia a fomentar la individualización de la enseñanza y los aprendizajes, se manifiesta a favor de un enfoque de competencias laborales, refuerza el vocacionalismo, la deshomologación de los salarios del personal académico y la acreditación de sus funciones suscrita y validada por agencias externas (Didriksson, 2002, p. 368).

Lograr la consolidación de cuerpos académicos y áreas de conocimiento que realicen actividades de innovación académica, de generación y aplicación del conocimiento que resuelvan los problemas del entorno económico y social. El sistema de aprendizaje del nuevo modelo de educación superior, ciencia y tecnología debe apoyarse, aunque no necesariamente girar alrededor del uso intensivo de las tecnologías de la comunicación y la información (TICs). Las características de los perfiles de los egresados serán certificadas por organismos externos. La certificación profesional y la acreditación institucional con los estándares internacionales del ejercicio profesional impulsarán una cultura de la planeación colegiada y de la autoevaluación.

Los escenarios cuantitativos de matrícula, de titulación, de cobertura y la problemática en general de la educación superior para el año 2020, están determinados por las condiciones de desigualdad, inequidad e injusticia social. Se requiere de una expansión del sistema educativo así como de iniciativas gubernamentales y de otros sectores de la economía y de la sociedad, para favorecer el ingreso de la demanda social y ampliar la cobertura del sistema. Se tiene que plantear que el esfuerzo por realizar deberá concentrarse en relación con el cambio de planes de estudio, sus tiempos, los métodos de enseñanza y técnicas; debe atenderse esencialmente el cambio de los contenidos mismos de las disciplinas. Para los próximos años permanece como un desafío imperativo homologar las equivalencias de los planes de estudio en educación superior, de tal forma que se logren acuerdos entre los sistemas tecnológicos y universitarios.

La ANUIES (1999) recomienda en los próximos años lograr un sistema abierto de educación superior en contra de uno cerrado, la creación de redes institucionales, el uso de las tecnologías de la información y la comunicación para la formación de la universidad virtual, el 
otorgamiento de personalidad jurídica propia a las agencias evaluadoras, acreditadoras y certificadoras. Las reformas en los modos del pensar académico, Lanz (2002) las denomina como reformas de la tercera generación porque se gestan en los cuerpos académicos al interior de las IES y al exterior forman redes interinstitucionales de generación, investigación y aplicación del conocimiento. No obstante, los procesos de generación, investigación y aplicación del conocimiento toman sentido con la vinculación con los beneficiarios.

\section{Vinculación}

La vinculación Estado-IES-empresas-sociedad es un requisito indispensable para complementar los esfuerzos de atención a la demanda de los servicios de educación superior que plantea el crecimiento demográfico, de tal forma que se puedan crear y desarrollar profesiones necesarias a la realidad económica, social y política. La vinculación de la educación, la ciencia y la tecnología con los sectores productivos y sociales tendrá que fortalecerse. Los procesos de vinculación de las IES tienen que concebirse en forma abierta, dinámica y circular con los sectores productivos y sociales, de tal forma que se faciliten los flujos de información, comunicación, transferencia de recursos, conocimientos, tecnología, etc.

El desarrollo de proyectos de investigación, transferencia de tecnología e investigación tecnológica tiene que ser una responsabilidad compartida por fondos financieros mixtos provenientes de los agentes y actores que más se benefician: empresas, Estado y sociedad mexicana. Se deben impulsar e incrementar los niveles de investigación y de postgrado mediante la formación investigadora, docente, divulgadora de la cultura, con capacidad para propiciar el aprendizaje profundo, la generación del conocimiento, la solución de problemas en distintos campos científicos y tecnológicos de aplicación, prevista en las actuales políticas educativas. Una propuesta para motivar la participación del sector privado es la creación de programas donde los servicios de educación superior ofrezcan beneficios a los empresarios que atraiga inversión. El dinamismo del sector privado podría aliviar la presión de la creciente demanda, aunque con niveles disímiles y muy discutibles en la calidad del servicio ofrecido.

Aprovechando las ventajas que ofrece una sociedad de información y conocimiento, la movilidad internacional académica, investigadora y estudiantil potenciará el intercambio de experiencias, conocimientos, habilidades, valores y actitudes que ampliarán los horizontes de la formación profesional. Igualmente es importante que se fomenten programas de 
vinculación con las empresas multinacionales y globales, no solamente para realimentar los planes y programas de estudio, sino también para facilitar la inmersión laboral de los egresados. Las innovaciones institucionales para apoyar las funciones sustantivas de las IES requerirán de mejores estructuras para ampliar los servicios de los centros de negocios, programas de incubación empresarial, etc. Sin olvidar la vinculación con los sectores social y político a través de programas tales como las ciberbolsas de trabajo, apoyo a grupos en exclusión social y marginada, participación en proyectos específicos de los tres niveles de gobierno para la promoción y el desarrollo económico regional, etc.

A continuación se analiza el problema toral de la educación superior en México para los próximos años, su financiamiento.

\section{Finanzas}

El problema total de la educación superior ha sido, es y seguirá siendo el financiamiento y la planeación e instrumentalización financiera que implica la disponibilidad de recursos. Las IES asumirán compromisos de transparencia y rendición de cuentas sobre el ejercicio y operación de las finanzas, ya sea de procedencia pública o privada, en una lógica de complementación de recursos interinstitucionales. Las IES deberán estar más sometidas a la rendición de cuentas, auditorías y al escrutinio del uso de los recursos financieros por las autoridades educativas locales o estatales, a fin de evitar la centralización de estas funciones y por tanto, su mal manejo. Se presume que en un futuro aumentará la presión para que las propias instituciones generen ingresos complementarios, debido a la insuficiencia de recursos financiaros para atender el crecimiento de la demanda.

El Plan Nacional de Desarrollo (2006-2012) es muy escueto para señalar las posibles fuentes de financiamiento y los recursos necesarios para la educación, la investigación y la innovación tecnológica. Debido a los altos costos de la educación superior e investigación científica y tecnológica de calidad, los nuevos esquemas de financiamiento tendrán repercusiones serias en las relaciones interinstitucionales entre las IES y las autoridades gubernamentales. Estas repercusiones se verán reflejadas en la reducción de subsidios y asignaciones presupuestales, en una mayor eficiencia de ejercicio del gasto y los recursos disponibles. El ordenamiento institucional sujeta a las IES a procedimientos de vigilancia a distancia y autonomía regulada (Ibarra, 2001) con la finalidad de que las IES obtengan una identidad nueva más de acuerdo con su funcionalidad social como una moderna corporación 
social centrada en la producción de profesionales y generación y aplicación de conocimientos científicos y tecnológicos requeridos por las nuevas formas de producción del mercado global y las nuevas formas de relaciones requeridas por la sociedad global (Rubio, 2006).

Se hace urgente una reconfiguración de las identidades académicas de las IES y de los sujetos académicos, de tal forma y magnitud que abandonen su actual posicionamiento de meros agentes económicos productores de profesionales y conocimientos, para que recuperen su posición de instituciones que tienen referentes culturales, científicos, tecnológicos, de desarrollo social, legitimadoras de procesos sociales y del Estado mexicano, etc. La nueva identidad de los sujetos académicos facilita las prácticas transdisciplinarias e interinstitucionales para la formación de redes y cuerpos académicos que comparten objetivos en la generación, difusión, transferencia y aplicación de conocimientos científicos, tecnológicos y culturales.

De acuerdo a Ibarra Colado (2007), el modo de racionalidad neoliberal del diseño de la nueva universidad se delimita en 5 ejes: Un estado auditor con mecanismos de vigilancia a distancia en la efectividad de los resultados, el fomento de nuevas formas de financiamiento que incluyen programas de vinculación universidad - mercado - Estado, la diversificación y estratificación del Sistema Nacional de Educación Superior, modernización administrativa y la evaluación del desempeño académico con programas de remuneración individual a partir de indicadores de productividad. La mejora en la racionalidad para asignar los recursos financieros a las diversas instituciones de la educación pública es determinante para abandonar viejas prácticas de negociaciones interinstitucionales articuladas en los factores políticos - partidistas más que en una racionalidad centrada en resultados académicos. Por lo tanto, en los próximos años se pretende incrementar la calidad de los procesos de transparencia y rendición de cuentas de las responsabilidades sustantivas de las IES.

Las IES tienen que asumir acciones concretas para incrementar la transparencia y rendición de cuentas en el ejercicio y operación de fondos financieros públicos en forma articulada con su autonomía universitaria y demás componentes de los sistemas de educación superior, de tal forma que se propicie la colaboración complementariedad institucional. El Estado debe considerar la inversión en educación superior e investigación científica como inversión social. 
Las crecientes restricciones presupuestarias que presionan financieramente a las instituciones de educación superior en México, han impactado fuertemente la calidad en la entrega de los servicios educativos en el sector público. La fuerte carga fiscal que la educación superior representa para el Estado mexicano que no puede satisfacer todas las expectativas de formación científica y tecnológica de la sociedad mexicana, ha dado lugar a la transferencia de esa responsabilidad social al sector privado, no sin dificultades por las enormes inversiones de infraestructura que se requieren para proveer los servicios educativos con eficiencia y eficacia. El crecimiento cuantitativo del sector privado en educación e investigación generará un mayor reclutamiento de las y los estudiantes de la clase alta ofreciéndoles un mayor posicionamiento de liderazgo, a pesar de la heterogeneidad. Muchas IES recorrerán caminos de reconversión de universidades públicas a privadas mediante mecanismos de mercado de sus productos, tales como los egresados, conocimientos y valores.

El punto crucial por atender en la generación, investigación y aplicación del conocimiento en el futuro inmediato es la creación de fuentes alternativas de financiamiento de tal forma que se reduzcan las presiones presupuestales del Estado mexicano para atender las demandas. Sin embargo, todas las soluciones apuntan a descargar estas responsabilidades del Estado en las organizaciones del mercado bajo estructuras de gobernanza que impliquen procesos de vinculación del tipo proveedor - cliente, que aunque altamente discutible y debatible, puede ser parte de la solución, mientras se perfecciona el modelo para alcanzar las connotaciones de atención a la ciudadanía. La educación superior requiere de un esfuerzo sostenido de gestión de recursos financieros para afrontar las responsabilidades en materia de formación profesional, preservación de la identidad y los valores de la cultura, las ciencias y las técnicas, generación del conocimiento, innovación tecnológica, transferencia de tecnología, etc. Transferir las responsabilidades de aportación financiera al sector privado solo resuelve en parte el complejo problema porque limita las oportunidades de movilidad social a quienes no la necesitan porque pueden pagar su formación profesional, por lo que es imprescindible que el Estado asuma su responsabilidad financiera bajo una perspectiva de largo alcance para fortalecer la educación superior en sectores sociales altamente competitivos que carecen de los recursos para hacerlo por sí mismos.

Si bien se debe pugnar por impulsar la responsabilidad del Estado en la educación superior, para atender la demanda educativa en los próximos 13 años es necesario que los servicios 
educativos se expresen en términos de oferta educativa y demanda educativa en un mercado regulado, de recursos e insumos educativos para apuntalar a una sociedad del conocimiento y la información. La sociedad del conocimiento y la adopción del paradigma de la educación permanente y para toda la vida son referentes del contexto educativo postulados por la UNESCO.

La aplicación de la inversión pública en la educación superior constituye una respuesta a las necesidades de la mayoría de mexicanas y mexicanos (Más del 66\% según datos de ANUIES, 1999) que no tienen la oportunidad de acceder a la educación superior a efecto de que todas las IES públicas y privadas tengan la infraestructura educativa y la planta académica idóneas para ofrecer una educación de calidad. Para lograrlo, se requiere aplicar nuevas modalidades de financiamiento de la educación superior pública que respondan adecuadamente en cobertura, pertinencia y calidad. Por otra parte aumentará la participación social responsable en el gasto educativo pero también para constituirse en auditores sociales, como mecanismos para exigir la transparencia y la rendición de cuentas que manejen directivos y académicos (personal docente e investigador) para una correcta aplicación de los recursos financieros.

La gestión de recursos financieros adquirirá relevancia en los próximos años, lo cual requerirá una mayor dosis de creatividad para allegarse de los recursos escasos, con nuevas acciones como por ejemplo explorar programas de vinculaciones con otras universidades y fundaciones que cuentan con bolsas de financiación para proyectos específicos de formación profesional, creación de conocimiento científico y tecnológico, programas de innovación tecnológica, transferencia de tecnología, apoyo a grupos discriminados para acceso a oportunidades educacionales, etc. En los próximos años se debe incrementar la capacidad de gestión para captar fondos de financiamiento, como por ejemplo, mediante la oferta de actividades de docencia, investigación, innovación, desarrollo y vinculación que puedan catalogarse como servicios con efectos multiplicadores que derramen los beneficios hacia estudiantes y personas investigadoras. Se esperaría que en el México del futuro, la iniciativa privada incrementara sus aportaciones, lo que haría a la ciudadanía mexicana menos dependiente de los avances que en esta materia se realicen en los países más avanzados.

La escasez de recursos financieros y la urgente necesidad de financiamiento de las actividades de la educación superior, sobre todo en lo concerniente a infraestructura y 
equipamiento educativo, propiciarán la generación de recursos por otras fuentes tales como la creación de fideicomisos, créditos institucionales e ingresos propios por la vía de venta de servicios. Por otro lado, también es sumamente importante que se considere que el incremento en las percepciones vía aumento de sueldos de docentes repercuta en una mejor calidad de la educación. Hay que acotar que las remuneraciones de docentes, sin embargo, deben ser de acuerdo con el desarrollo y crecimiento de la economía nacional y compatible con los resultados del desempeño. Finalmente, este trabajo analiza la prospectiva de la educación superior en México en función de los resultados esperados de los procesos de evaluación integral del proceso educativo.

\section{Evaluación integral del proceso educativo}

El actual Plan Nacional de Desarrollo (2006-2012) propone la creación del Sistema Nacional de Evaluación Educativa en la educación media superior y superior y en la medición del desempeño docente. En educación superior, ciencia y tecnología es una prioridad en los próximos años establecer un sistema de evaluación integral de la educación más centrada en la evaluación de la totalidad del proceso educativo, incluyendo sus resultados. La evaluación integral del desempeño del proceso educativo ha desplazado al enfoque más centrado en la planeación para ejercer un mayor control de la vigilancia a distancia de las actividades académicas de las IES. La tendencia en los procesos de evaluación se mantendrá en los mecanismos de la evaluación diagnóstica de programas, evaluación de insumos, resultados de los procesos educativos y la acreditación formal, por organismos independientes del sistema pero con reconocimiento de las autoridades educativas. La evaluación de la calidad institucional propiciará la innovación académica.

La evaluación por resultados con fundamento en un modelo de financiamiento se centra en criterios de desempeño y programas integrales de fortalecimiento institucional. La evaluación del desempeño individual de los diferentes actores involucrados, tales como personal investigativo, docente, estudiantil, administrativo y de mantenimiento, directivo, etc., exige cambios de actitudes y comportamientos más de acuerdo con el despliegue de una racionalidad económica que solo beneficia a los mismos actores individualmente y se pierden valores solidarios.

Para salir de la actual crisis de la educación superior en México manifiesta en los resultados de aprovechamiento académico en las evaluaciones de la Organización de Estados para la 
Cooperación y Desarrollo (OECD), es menester que los académicos salven los obstáculos de las trampas tendidas por la orientación a evaluar la cantidad más que la calidad de los procesos de evaluación del desempeño y productividad individual bajo el esquema de la profesionalización que ha implicado cambios en los comportamientos y modos de ser y actuar. Ya es tiempo de recuperar lo que se ha perdido: abandonar la postura del trabajo universitario por una vuelta a la vocación académica que bien describe Ibarra Colado (2007); de abandonar la destructiva competencia, por valores más solidarios, la práctica del individualismo egoísta y posesivo por los valores institucionales, etc., como por ejemplo en el caso de la beca al desempeño académico, que ha logrado lo que Urbano et al (2006) describe como la reconstitución de comportamientos y modos de ser que hacen de los actores de la universidad sujetos muy distintos y cuya nueva identidad está sujeta al rendimiento en la productividad, la capitulación y la información. Se requiere una nueva reconfiguración de las identidades de los sujetos académicos para solventar la crisis moral y ética propiciada por los principios de la racionalidad instrumental del modelo de educación superior centrado en el individualismo y la competencia, por otro modelo que permita el desarrollo solidario más acorde con los valores del desarrollo humano y más centrado en el proyecto institucional de las IES como garantes de la sociedad en plena coordinación con los valores del mercado y del Estado.

Este sistema debe evaluar el desempeño de todos los actores involucrados en los procesos educativos, incluyendo al Estado, la comunidad, las funcionarias y los funcionarios educativos, docentes, grupo investigador, padres y madres de familia, instituciones de apoyo, estudiantes, etc. Igualmente, el sistema de evaluación integral del proceso educativo debe evaluar las instituciones, infraestructura, recursos financieros y materiales, recursos y medios didácticos, etc.

Para lograr una mayor eficiencia en los recursos se hace necesario implementar sistemas de evaluación, acreditación y rendición de cuentas de la aplicación de recursos. Se requieren políticas, estrategias, normatividad e indicadores de desempeño de la calidad académica y de atención a las demandas del entorno y del mercado laboral. Es además importante dar difusión a los resultados de las evaluaciones, asegurar la realimentación a los actores involucrados en los procesos educativos para establecer las medidas correctivas pertinentes. 


\section{Tareas pendientes}

Existe ya una conciencia de la crisis de la educación superior generada por problemas de eficiencia, eficacia y cumplimiento de sus funciones sustantivas por parte de todos los actores, internos y externos involucrados. Las reformas sobre calidad, cobertura y pertinencia en el ámbito de la modernización plantean nuevos desafíos y nuevas tareas bajo una tendencia de multidireccionalidad como el fundamento para la transformación de la función central de una formación transdisciplinaria de profesionales más apropiada para promover el desarrollo social y económico acorde con el contexto de los tiempos contemporáneos.

Es en este sentido que las IES necesitan redefinirse relación con su entorno, replantearse su visión, misión y funciones (Gutiérrez, 2001, pp. 33-95). El rediseño y reconfiguración de las IES mediante procesos de transformación que permitan el libre flujo del pensamiento, ideologías, información y conocimientos desde la perspectiva del análisis crítico, es un desafío ineludible de los tiempos por venir. Los procesos de transformación de la educación superior así orientados son como el sustrato que posibilita todo el desarrollo de las capacidades humanas para la creatividad e innovación que permitirán dar respuesta a la realidad compleja e incierta y facultará el logro de más altos niveles de crecimiento económico a la par de un desarrollo sustentable social y ambiental. El principal desafío imperativo de las IES en los próximos años será romper con la continuidad de las políticas educativas que se implementan con las reformas estructurales por mandato de intereses extraños al desarrollo sustentable de la sociedad mexicana. En este sentido, las propuestas están llenas de propósitos, por lo que se puede argumentar que se inscriben en una línea voluntarista y sin fundamentos en diagnósticos serios.

Uno de los principales retos es vencer los obstáculos y resistencias al cambio institucional capaz de eliminar las tendencias perturbadoras y perversas de la implementación del modelo neoliberal de educación superior centrado en la gobernabilidad del libre mercado. Esto conlleva a la exaltación del individualismo y procesos excluyentes de competitividad económica. Para revertir estas tendencias, la propuesta va en el sentido de fomentar procesos de generación, difusión, transferencia y aplicación de conocimientos mediante estructuras y comportamientos organizacionales centrados en la cooperación y la solidaridad. 
La orientación multidireccional de las transformaciones educativas implican comprender la transformación en las formas de gobierno, en la administración académica, en la evaluación de la calidad de los procesos y resultados, en la responsabilidad compartida y cooperación de unidades, en el compromiso por la construcción de un proyecto alternativo de sociedad, en la democratización de sus estructuras de representación, en la formulación e implementación de sus modos de financiamiento, en la integración del sistema universitario con el resto del sistema educativo superior, etc. (Luengo, 2003, p. 46)

Los procesos de transformación institucional deben implicar estrategias impulsadas por los liderazgos académicos en las IES mediante procesos de consenso y autorregulación que involucren a las comunidades académicas en propuestas de cambio de gran escala y de largo alcance, lo cual requiere la integración y consolidación de cuerpos académicos colegiados o colectivos. Las tareas pendientes propuestas por De la Fuente (2007) para avanzar en la sociedad del conocimiento son: mayores inversiones en educación, investigación y desarrollo, formar una red de universidades de clase mundial, incrementar los niveles académicos de la población económicamente activa e importar conocimientos y no solo exportarlos. Las reformas universitarias deben ampliarse a todos los ámbitos de las actividades sustantivas, la calidad en los procesos de generación, investigación y aplicación del conocimiento, los proyectos de vinculación con los sectores productivos y sociales y la calidad de la infraestructura y uso de tecnologías de la información y la comunicación.

La formación profesional del futuro debe ser capaz de conjugar los saberes pragmáticos y empíricos con los conocimientos teóricos metodológicos. La reorganización de los saberes y sus interrelaciones disciplinarias y transdisciplinarias, su relación curricular y los procesos de enseñanza-aprendizaje, etc., son cuestiones urgentes que la transformación de la gestión académica institucional debe atender. La educación superior tiene entre sus principales retos el desarrollo de contenidos educativos de planes y programas de estudio desde la transdisciplinariedad, la complejidad y la incertidumbre, las estrategias metodológicas, las nuevas formas de organización y gobierno, la creación de alianzas estratégicas para la complementariedad de los recursos, etc. En las nuevas formas de organización se pretende administrar los procesos de transformación innovadora de la educación superior para lograr mayor eficiencia en la toma de decisiones, con estructuras descentralizadas que permitan y promuevan una mayor delegación de la autoridad en las funciones sustantivas, pero también 
una mayor participación e integración de los diferentes actores articulados en redes de cooperación académica y científica.

Los procesos de transformación de las IES en México enfrentarán como uno de los principales retos dar respuesta de una forma más responsable a las demandas de los sectores productivos y sociales. Los desafíos de la realidad educativa requieren de la cooperación y la solidaridad como los fundamentos para evitar la marginación, fragmentación y polarización del conocimiento. Se requiere asumir un discurso crítico sobre los alcances de las funciones del nuevo modelo de las IES bajo una orientación prioritaria a los requerimientos del mercado, subestimando las necesidades sociales y las del Estado mexicano.

\section{Referencias}

Acosta Silva, Alberto. (2000). Estado, políticas y universidades en una período de transición. México: Universidad de Guadalajara/Fondo de Cultura Económica.

Altbch, Philip. (2002). Educación superior privada. México: UNAM/Grupo editorial Porrúa.

Analítica Consultores. (2006, marzo). México Visión 2030: Prospectiva de Largo Plazo Grupo de Enfoque: Educación 2 (Síntesis de resultados). Foro Consultivo Científico y Tecnológico. Consejo Nacional de Ciencia y Tecnología. México, DF: Analítica Consultores.

Asociación Nacional de Universidades e Instituciones de Educación Superior (ANUIES) México. (1999) La educación superior en el siglo XXI. Líneas estratégicas de desarrollo. Asociación Nacional de Universidades e Instituciones de Educación Superior. México.

Barnett, Ronald. (2002) Claves para entender la universidad, en una era de supercomplejidad. Girona: Ediciones Pomares.

Casanova Cardiel, Hugo. (2007, Septiembre). Universidad y educación pública: Una responsabilidad de Estado. Este País, (198), pp. 35-38.

Casanova Cardiel, Hugo. (2002). La universidad hoy: idea y tendencias de cambio. En Muñoz, Humberto, Universidad: política y cambio institucional (pp. 31-35). México: CESU-UNAM/Porrúa.

Congreso Internacional. (1997). Quelle université pour domain? Veers una évolution transdisciplinaire de l'université, Locarno, Suiza.

De la Fuente, Juan Ramón. (2007). “Universidad y desarrollo”, Este País, 197, Agosto 2007, Páginas 12-14. 
Didriksson T., Axel y Herrera, Alma. (Coord.), La transformación de la universidad mexicana: diez estudios de caso en la transición. México: Universidad Autónoma de Zacatecas/Grupo Editorial Porrúa.

Gutiérrez, Alfredo. (2001). El conocimiento empieza por nosotros: de la autocrítica a la univermoción. En Luengo, Enrique, Educación, mundialización y democracia: un circuito crítico. México: UNLA/ITESO/UIA/UVM/IIPC/UNESCO.

Herrera, Alma. (2002). El cambio en la década de los noventa: estudio comparado de diez universidades públicas de México. En Didrikson T., Axel y Alma Herrera (Coord.), La transformación de la universidad mexicana: diez estudios de caso en la transición (pp. 9-10). México: Universidad Autónoma de Zacatecas/Grupo Editorial Porrúa.

Ibarra Colado, Eduardo. (2007,16 de noviembre). Reconfiguración institucional de la "Nueva Universidad" en México. Gaceta Ide@s CONCYTEG, Año 2, (28).

Ibarra Colado, Eduardo. (2002, enero-abril). La "nueva universidad" en México: transformaciones recientes y perspectivas. Revista Mexicana de Investigación Educativa, 7 (14), 75-105.

Ibarra Colado, Eduardo. (2001). La universidad en México hoy: Gubernamentalidad y modernización ( $\mathrm{N}^{\circ}$ 16). México: DGEP-UNAM/FCPyS-UNAM/UAMIztapalapa/ANUIES, Col. Posgrado.

Instituto Nacional de Estadística, Geografía e Informática (INEGI) - México. (2000). Censo Nacional de Población. Ags. Cal., México: INEGl.

Lanz, Rigoberto. (2003, marzo). ¿Qué quiere decir "reforma universitaria"?: lo que quieren los que quieren reformas. Caracas: mimeo.

Lanz, Rigoberto. (2002, octubre). ¿Quién teme a las reformas? Caracas: mimeo.

Lloréns Báez, Luis. (s.f.). Viabilidad de un sistema de educación superior en México: Comentarios al documento La educación superior hacia el Siglo XXI. México: [s.n.].

Luengo González Enrique. (2003, 5 y 6 de junio). Tendencias de la educación superior en México: Una lectura desde la perspectiva de la complejidad. Trabajo elaborado para el Seminario sobre Reformas de la Educación Superior en América Latina y el Caribe, Bogotá, Colombia. Instituto Internacional de la UNESCO para la Educación Superior en América Latina y el Caribe (IESACC) y la Asociación Colombiana de Universidades (ASCUN).

Meyer, John W. y Rowan, Brian. (1999). Organizaciones institucionalizadas: La estructura formal como mito y ceremonia. En Powell, W.W. y DiMaggio P. J. (comps.). El nuevo institucionalismo en el análisis organizacional. México. Fondo de Cultura Económica /Colegio Nacional de Ciencias Políticas y Administración Pública/UAEM.

Morin, Edgar. (1999a). La cabeza bien puesta: repensar la reforma, reformar el pensamiento. Buenos Aires: Nueva Visión. 
Morin, Edgar. (1999b). Los siete saberes necesarios para la educación del futuro. París: UNESCO.

Morin, Edgar. (1999c). Sobre la interdisciplinariedad. En Boletín del Centre Internacional de Recherches et Etudes Transdisciplianires (CIRET), (2), París.

Nicolescu, Basarab. (1997). The transdisciplinary evolution of university condition for sustainable developlement. Ponencia presentada en el Congreso Universities responsabilities to society, Bankok.

Observatorio Ciudadano de la Educación. (2007, Septiembre). ¿Un nuevo modelo educativo para el México del siglo XXI? La educación que propone el SNTE. Este País, (198), 3034.

Observatorio Ciudadano de la Educación. (2007b, Agosto). La "transformación educativa" según el Plan Nacional de Desarrollo 2007-2012. Este País, (107), 34-39.

Rodríguez, Roberto. (1998). The modernization of higher education in Mexico: an agenda for discusión. México: Centro de Estudios sobre la Universidad, Coordinación de Humanidades, Universidad Autónoma de México.

Rubio Oca, Julio. (Coord.). (2006). La política educativa y la educación superior en México. 1995-2006: Un balance. México: SEP/Fondo de Cultura económica.

Urbano Vidales, Guillermina; Aguilar Sahún, Guillermo y Rubio Oca, Julio. (2006). Programa de mejoramiento del profesorado. Un primer análisis de su operación e Impactos en el proceso de fortalecimiento académico de las universidades públicas, México: Secretaría de Educación Pública. 